\title{
On Inclusion Relations between Some Sequence Spaces
}

\author{
R. Çolak, Ç. A. Bektaş, H. Altınok, and S. Ercan \\ Department of Mathematics, Firat University, 23119 Elâziğ, Turkey \\ Correspondence should be addressed to Ç. A. Bektaş; cbektas@firat.edu.tr
}

Received 15 April 2016; Accepted 10 July 2016

Academic Editor: Julien Salomon

Copyright (C) 2016 R. Çolak et al. This is an open access article distributed under the Creative Commons Attribution License, which permits unrestricted use, distribution, and reproduction in any medium, provided the original work is properly cited.

We determine the relations between the classes $\widehat{S}_{\lambda}$ of almost $\lambda$-statistically convergent sequences and the relations between the classes $[\widehat{V}, \lambda]$ of strongly almost $(V, \lambda)$-summable sequences for various sequences $\lambda$, $\mu$ in the class $\Lambda$. Furthermore we also give the relations between the classes $\widehat{S}_{\lambda}$ of almost $\lambda$-statistically convergent sequences and the classes $[\widehat{V}, \lambda]$ of strongly almost $(V, \lambda)$-summable sequences for various sequences $\lambda, \mu \in \Lambda$.

\section{Introduction}

A sequence $x=\left(x_{k}\right)$ of real (or complex) numbers is said to be statistically convergent to the number $L$ if for every $\varepsilon>0$

$$
\lim _{n \rightarrow \infty} \frac{1}{n}\left|\left\{k \leq n:\left|x_{k}-L\right| \geq \varepsilon\right\}\right|=0 .
$$

In this case, we write $S-\lim x=L$ or $x_{k} \rightarrow L(S)$ and $S$ denotes the set of all statistically convergent sequences.

A sequence $x=\left(x_{k}\right)$ of real (or complex) numbers is said to be almost statistically convergent to the number $L$ if for every $\varepsilon>0$

$$
\lim _{n \rightarrow \infty} \frac{1}{n}\left|\left\{k \leq n:\left|x_{k+m}-L\right| \geq \varepsilon\right\}\right|=0 \quad \text { uniformly in } m .
$$

In this case, we write $\widehat{S}-\lim x=L$ or $x_{k} \rightarrow L(\widehat{S})$ and $\widehat{S}$ denotes the set of all almost statistically convergent sequences [1].

Let $\lambda=\left(\lambda_{n}\right)$ be a nondecreasing sequence of positive real numbers tending to $\infty$ such that

$$
\lambda_{n+1} \leq \lambda_{n}+1, \quad \lambda_{1}=1 .
$$

The set of all such sequences will be denoted by $\Lambda$.

The generalized de la Vallée-Poussin mean is defined by

$$
t_{n}(x)=\frac{1}{\lambda_{n}} \sum_{k \in I_{n}} x_{k}
$$

where $I_{n}=\left[n-\lambda_{n}+1, n\right]$.
A sequence $x=\left(x_{k}\right)$ is said to be $(V, \lambda)$-summable to a number $L$ (see [2]) if

$$
t_{n}(x) \longrightarrow L \quad \text { as } n \longrightarrow \infty
$$

If $\lambda_{n}=n$ for each $n \in \mathbb{N}$, then $(V, \lambda)$-summability reduces to $(C, 1)$-summability.

We write

$[C, 1]$

$$
=\left\{x=\left(x_{k}\right): \lim _{n \rightarrow \infty} \frac{1}{n} \sum_{k=1}^{n}\left|x_{k}-L\right|=0 \text { for some } L\right\} \text {, }
$$

$[V, \lambda]$

$$
=\left\{x=\left(x_{k}\right): \lim _{n \rightarrow \infty} \frac{1}{\lambda_{n}} \sum_{k \in I_{n}}\left|x_{k}-L\right|=0 \text { for some } L\right\}
$$

for the sets of sequences $x=\left(x_{k}\right)$ which are strongly Cesàro summable and strongly $(V, \lambda)$-summable to $L$; that is, $x_{k} \rightarrow$ $L[C, 1]$ and $x_{k} \rightarrow L[V, \lambda]$, respectively. 
Savaš [1] defined the following sequence space:

$$
\begin{gathered}
{[\widehat{V}, \lambda]=\left\{x=\left(x_{k}\right): \lim _{n \rightarrow \infty} \frac{1}{\lambda_{n}} \sum_{k \in I_{n}}\left|x_{k+m}-L\right|\right.} \\
=0 \text { for some } L \text {, uniformly in } m\}
\end{gathered}
$$

for the sets of sequences $x=\left(x_{k}\right)$ which are strongly almost $(V, \lambda)$-summable to $L$; that is, $x_{k} \rightarrow L[V, \lambda]$. We will write $[\widehat{V}, \lambda]_{\infty}=[\widehat{V}, \lambda] \cap \ell_{\infty}$.

The $\lambda$-statistical convergence was introduced by Mursaleen in [3] as follows.

Let $\lambda=\left(\lambda_{n}\right) \in \Lambda$. A sequence $x=\left(x_{k}\right)$ is said to be $\lambda$ statistically convergent or $S_{\lambda}$-convergent to $L$ if for every $\varepsilon>0$

$$
\lim _{n \rightarrow \infty} \frac{1}{\lambda_{n}}\left|\left\{k \in I_{n}:\left|x_{k}-L\right| \geq \varepsilon\right\}\right|=0,
$$

where $I_{n}=\left[n-\lambda_{n}+1, n\right]$. In this case we write $S_{\lambda}-\lim x=L$ or $x_{k} \rightarrow L\left(S_{\lambda}\right)$, and $S_{\lambda}=\left\{x=\left(x_{k}\right): S_{\lambda}-\lim x=L\right.$ for some $\left.L\right\}$.

The sequence $x=\left(x_{k}\right)$ is said to be $\lambda$-almost statistically convergent if there is a complex number $L$ such that

$$
\lim _{n \rightarrow \infty} \frac{1}{\lambda_{n}}\left|\left\{k \in I_{n}:\left|x_{k+m}-L\right| \geq \varepsilon\right\}\right|=0
$$

uniformly in $m$.

In this case, we write $\widehat{S}_{\lambda}-\lim x=L$ or $x_{k} \rightarrow L\left(\widehat{S}_{\lambda}\right)$ and $\widehat{S}_{\lambda}$ denotes the set of all $\lambda$-almost statistically convergent sequences. If we choose $\lambda_{n}=n$ for all $n$, then $\lambda$-almost statistical convergence reduces to almost statistical convergence [1].

\section{Main Results}

Throughout the paper, unless stated otherwise, by "for all $n \in$ $\mathbb{N}_{n_{o}}$ " we mean "for all $n \in \mathbb{N}$ except finite numbers of positive integers" where $\mathbb{N}_{n_{o}}=\left\{n_{o}, n_{o}+1, n_{o}+2, \ldots\right\}$ for some $n_{o} \in$ $\mathbb{N}=\{1,2,3, \ldots\}$.

Theorem 1. Let $\lambda=\left(\lambda_{n}\right)$ and $\mu=\left(\mu_{n}\right)$ be two sequences in $\Lambda$ such that $\lambda_{n} \leq \mu_{n}$ for all $n \in \mathbb{N}_{n_{o}}$. Consider the following:

(i) If

$$
\liminf _{n \rightarrow \infty} \frac{\lambda_{n}}{\mu_{n}}>0
$$

then $\widehat{S}_{\mu} \subseteq \widehat{S}_{\lambda}$.

(ii) If

$$
\lim _{n \rightarrow \infty} \frac{\lambda_{n}}{\mu_{n}}=1
$$

then $\widehat{S}_{\lambda} \subseteq \widehat{S}_{\mu}$.
Proof. (i) Suppose that $\lambda_{n} \leq \mu_{n}$ for all $n \in \mathbb{N}_{n_{o}}$ and let (10) be satisfied. Then $I_{n} \subset J_{n}$ so that for $\varepsilon>0$ we may write

$$
\begin{aligned}
& \left|\left\{k \in J_{n}:\left|x_{k+m}-L\right| \geq \varepsilon\right\}\right| \\
& \quad \geq\left|\left\{k \in I_{n}:\left|x_{k+m}-L\right| \geq \varepsilon\right\}\right|
\end{aligned}
$$

and therefore we have

$$
\begin{aligned}
& \frac{1}{\mu_{n}}\left|\left\{k \in J_{n}:\left|x_{k+m}-L\right| \geq \varepsilon\right\}\right| \\
& \quad \geq \frac{\lambda_{n}}{\mu_{n}} \frac{1}{\lambda_{n}}\left|\left\{k \in I_{n}:\left|x_{k+m}-L\right| \geq \varepsilon\right\}\right|
\end{aligned}
$$

for all $n \in \mathbb{N}_{n_{o}}$, where $J_{n}=\left[n-\mu_{n}+1, n\right]$. Now taking the limit as $n \rightarrow \infty$ uniformly in $m$ in the last inequality and using (10) we get $x_{k} \rightarrow L\left(\widehat{S}_{\mu}\right) \Rightarrow x_{k} \rightarrow L\left(\widehat{S}_{\lambda}\right)$ so that $\widehat{S}_{\mu} \subseteq \widehat{S}_{\lambda}$.

(ii) Let $\left(x_{k}\right) \in \widehat{S}_{\lambda}$ and (11) be satisfied. Since $I_{n} \subset J_{n}$, for $\varepsilon>0$, we may write

$$
\begin{aligned}
\frac{1}{\mu_{n}}\left|\left\{k \in J_{n}:\left|x_{k+m}-L\right| \geq \varepsilon\right\}\right| \\
=\frac{1}{\mu_{n}}\left|\left\{n-\mu_{n}+1 \leq k \leq n-\lambda_{n}:\left|x_{k+m}-L\right| \geq \varepsilon\right\}\right| \\
\quad+\frac{1}{\mu_{n}}\left|\left\{k \in I_{n}:\left|x_{k+m}-L\right| \geq \varepsilon\right\}\right| \\
\leq \frac{\mu_{n}-\lambda_{n}}{\mu_{n}}+\frac{1}{\lambda_{n}}\left|\left\{k \in I_{n}:\left|x_{k+m}-L\right| \geq \varepsilon\right\}\right| \\
\leq\left(1-\frac{\lambda_{n}}{\mu_{n}}\right)+\frac{1}{\lambda_{n}}\left|\left\{k \in I_{n}:\left|x_{k+m}-L\right| \geq \varepsilon\right\}\right|
\end{aligned}
$$

for all $n \in \mathbb{N}_{n_{0}}$. Since $\lim _{n}\left(\lambda_{n} / \mu_{n}\right)=1$ by (11) and since $x=$ $\left(x_{k}\right) \in \widehat{S}_{\lambda}$ the first term and second term of right hand side of above inequality tend to 0 as $n \rightarrow \infty$ uniformly in $m$. This implies that $\left(1 / \mu_{n}\right)\left|\left\{k \in J_{n}:\left|x_{k+m}-L\right| \geq \varepsilon\right\}\right| \rightarrow 0$ as $n \rightarrow \infty$ uniformly in $m$. Therefore $\widehat{S}_{\lambda} \subseteq \widehat{S}_{\mu}$.

From Theorem 1 we have the following result.

Corollary 2. Let $\lambda=\left(\lambda_{n}\right)$ and $\mu=\left(\mu_{n}\right)$ be two sequences in $\Lambda$ such that $\lambda_{n} \leq \mu_{n}$ for all $n \in \mathbb{N}_{n_{o}}$. If (11) holds then $\widehat{S}_{\lambda}=\widehat{S}_{\mu}$.

If we take $\mu=\left(\mu_{n}\right)=(n)$ in Corollary 2 we have the following result.

Corollary 3. Let $\lambda=\left(\lambda_{n}\right) \in \Lambda$. If $\lim _{n}\left(\lambda_{n} / n\right)=1$ then we have $\widehat{S}_{\lambda}=\widehat{S}$.

Theorem 4. Let $\lambda=\left(\lambda_{n}\right)$ and $\mu=\left(\mu_{n}\right) \in \Lambda$ and suppose that $\lambda_{n} \leq \mu_{n}$ for all $n \in \mathbb{N}_{n_{0}}$. Consider the following:

(i) If (10) holds then $[\widehat{V}, \mu] \subseteq[\widehat{V}, \lambda]$.

(ii) If (11) holds then $[\widehat{V}, \lambda]_{\infty} \subseteq[\widehat{V}, \mu]$. 
Proof. (i) Suppose that $\lambda_{n} \leq \mu_{n}$ for all $n \in \mathbb{N}_{n_{o}}$. Then $I_{n} \subseteq J_{n}$ so that we may write

$$
\frac{1}{\mu_{n}} \sum_{k \in J_{n}}\left|x_{k+m}-L\right| \geq \frac{1}{\mu_{n}} \sum_{k \in I_{n}}\left|x_{k+m}-L\right|
$$

for all $n \in \mathbb{N}_{n_{o}}$. This gives that

$$
\frac{1}{\mu_{n}} \sum_{k \in J_{n}}\left|x_{k+m}-L\right| \geq \frac{\lambda_{n}}{\mu_{n}} \frac{1}{\lambda_{n}} \sum_{k \in I_{n}}\left|x_{k+m}-L\right| .
$$

Then taking limit as $n \rightarrow \infty$, uniformly in $m$ in the last inequality, and using (10) we obtain $x_{k} \rightarrow L[\widehat{V}, \mu] \Rightarrow x_{k} \rightarrow$ $L[\widehat{V}, \lambda]$.

(ii) Let $x=\left(x_{k}\right) \in[\widehat{V}, \lambda]_{\infty}$ be any sequence. Suppose that $x_{k} \rightarrow L[\widehat{V}, \mu]$ and that (11) holds. Since $x=\left(x_{k}\right) \in l_{\infty}$ then there exists some $M>0$ such that $\left|x_{k+m}-L\right| \leq M$ for all $k$ and $m$. Since $\lambda_{n} \leq \mu_{n}$ so that $1 / \mu_{n} \leq 1 / \lambda_{n}$, and $I_{n} \subset J_{n}$ for all $n \in \mathbb{N}_{n_{o}}$, we may write

$$
\begin{aligned}
\frac{1}{\mu_{n}} \sum_{k \in J_{n}}\left|x_{k+m}-L\right|= & \frac{1}{\mu_{n}} \sum_{k \in J_{n}-I_{n}}\left|x_{k+m}-L\right| \\
& +\frac{1}{\mu_{n}} \sum_{k \in I_{n}}\left|x_{k+m}-L\right| \\
\leq & \frac{\mu_{n}-\lambda_{n}}{\mu_{n}} M+\frac{1}{\mu_{n}} \sum_{k \in I_{n}}\left|x_{k+m}-L\right| \\
& \leq\left(1-\frac{\lambda_{n}}{\mu_{n}}\right) M+\frac{1}{\lambda_{n}} \sum_{k \in I_{n}}\left|x_{k+m}-L\right|
\end{aligned}
$$

for every $n \in \mathbb{N}_{n_{o}}$. Since $\lim _{n}\left(\lambda_{n} / \mu_{n}\right)=1$ by (11) and since $x_{k} \rightarrow L[\widehat{V}, \lambda]$ the first term and the second term of right hand side of above inequality tend to 0 as $n \rightarrow \infty$, uniformly in $m$ (note that $1-\lambda_{n} / \mu_{n} \geq 0$ for all $n \in \mathbb{N}_{n_{0}}$ ). Then we get $x_{k} \rightarrow L[\widehat{V}, \lambda] \Rightarrow x_{k} \rightarrow L[\widehat{V}, \mu]$. Since $x=\left(x_{k}\right) \in[\widehat{V}, \lambda]_{\infty}$ is an arbitrary sequence we obtain $[\widehat{V}, \lambda]_{\infty} \subseteq[\widehat{V}, \mu]$.

Since clearly (11) implies (10) from Theorem 4 we have the following result.

Corollary 5. Let $\lambda, \mu \in \Lambda$ such that $\lambda_{n} \leq \mu_{n}$ for all $n \in \mathbb{N}_{n_{o}}$. If (11) holds then $[\widehat{V}, \lambda]_{\infty}=[\widehat{V}, \mu]_{\infty}$.

Theorem 6. Let $\lambda, \mu \in \Lambda$ such that $\lambda_{n} \leq \mu_{n}$ for all $n \in \mathbb{N}_{n_{o}}$. Consider the following:

(i) If (10) holds then

$$
x_{k} \longrightarrow L[\widehat{V}, \mu] \Longrightarrow x_{k} \longrightarrow L\left(\widehat{S}_{\lambda}\right)
$$

and the inclusion $[\widehat{V}, \mu] \subset \widehat{S}_{\lambda}$ holds for some $\lambda, \mu \in \Lambda$.

(ii) If $\left(x_{k}\right) \in \ell_{\infty}$ and $x_{k} \rightarrow L\left(\widehat{S}_{\lambda}\right)$ then $x_{k} \rightarrow L[\widehat{V}, \mu]$, whenever (11) holds.

(iii) If (11) holds then $\ell_{\infty} \cap \widehat{S}_{\lambda}=[\widehat{V}, \mu]_{\infty}$.
Proof. (i) Let $\varepsilon>0$ be given and let $x_{k} \rightarrow L[\widehat{V}, \mu]$. Now for every $\varepsilon>0$ we may write

$$
\begin{aligned}
\sum_{k \in J_{n}}\left|x_{k+m}-L\right| & \geq \sum_{k \in I_{n}}\left|x_{k+m}-L\right| \\
& \geq \sum_{\substack{k \in I_{n} \\
\left|x_{k+m}-L\right| \geq \varepsilon}}\left|x_{k+m}-L\right| \\
& \geq \varepsilon\left|\left\{k \in I_{n}:\left|x_{k+m}-L\right| \geq \varepsilon\right\}\right|
\end{aligned}
$$

so that

$$
\begin{aligned}
& \frac{1}{\mu_{n}} \sum_{k \in J_{n}}\left|x_{k+m}-L\right| \\
& \quad \geq \frac{\lambda_{n}}{\mu_{n}} \frac{1}{\lambda_{n}}\left|\left\{k \in I_{n}:\left|x_{k+m}-L\right| \geq \varepsilon\right\}\right| \varepsilon
\end{aligned}
$$

for all $n \in \mathbb{N}_{n_{0}}$. Then taking limit as $n \rightarrow \infty$, uniformly in $m$ in the last inequality, and using (10) we obtain that $x_{k} \rightarrow$ $L\left(\widehat{S}_{\lambda}\right)$ whenever $x_{k} \rightarrow L[\widehat{V}, \mu]$. Since $x=\left(x_{k}\right) \in[\widehat{V}, \mu]$ is an arbitrary sequence we obtain that $[\widehat{V}, \mu] \subset \widehat{S}_{\lambda}$.

(ii) Suppose that $x_{k} \rightarrow L\left(\widehat{S}_{\lambda}\right)$ and $x=\left(x_{k}\right) \in \ell_{\infty}$. Then there exists some $M>0$ such that $\left|x_{k+m}-L\right| \leq M$ for all $k$ and $m$. Since $1 / \mu_{n} \leq 1 / \lambda_{n}$, then for every $\varepsilon>0$ we may write

$$
\begin{aligned}
& \frac{1}{\mu_{n}} \sum_{k \in J_{n}}\left|x_{k+m}-L\right|= \frac{1}{\mu_{n}} \sum_{k \in J_{n}-I_{n}}\left|x_{k+m}-L\right| \\
&+\frac{1}{\mu_{n}} \sum_{k \in I_{n}}\left|x_{k+m}-L\right| \\
& \leq \frac{\mu_{n}-\lambda_{n}}{\mu_{n}} M+\frac{1}{\mu_{n}} \sum_{k \in I_{n}}\left|x_{k+m}-L\right| \\
& \leq\left(1-\frac{\lambda_{n}}{\mu_{n}}\right) M+\frac{1}{\lambda_{n}} \sum_{k \in I_{n}}\left|x_{k+m}-L\right| \\
& \leq\left(1-\frac{\lambda_{n}}{\mu_{n}}\right) M \\
&+\frac{1}{\lambda_{n}} \sum_{k \in I_{n}}\left|x_{k+m}-L\right| \\
&+\frac{M}{\lambda_{n}}\left|\left\{k \in I_{n}:\left|x_{k+m}-L\right| \geq \varepsilon\right\}\right| \\
&+\varepsilon \sum_{k \in I_{n}}\left|x_{k+m}-L\right| \\
& \lambda_{k+m}-L \mid<\varepsilon \\
& \mu_{n}
\end{aligned}
$$


for all $n \in \mathbb{N}_{n_{o}}$. Using (11) we obtain that $x_{k} \rightarrow L[\widehat{V}, \mu]$ whenever $x_{k} \rightarrow L\left(\widehat{S}_{\lambda}\right)$. Since $x=\left(x_{k}\right) \in \ell_{\infty} \cap \widehat{S}_{\lambda}$ is an arbitrary sequence we obtain $\ell_{\infty} \cap \widehat{S}_{\lambda} \subseteq[\widehat{V}, \mu]$.

(iii) The proof follows from (i) and (ii), so we omit it.

From Theorem 1(i) and Theorem 6(i) we obtain the following result.

Corollary 7. If $\liminf \operatorname{in}_{n \rightarrow \infty}\left(\lambda_{n} / \mu_{n}\right)>0$ then $\widehat{S}_{\mu} \cap[\widehat{V}, \mu] \subset \widehat{S}_{\lambda}$.

If we take $\mu_{n}=n$ for all $n$ in Theorem 6 then we have the following results, because $\lim _{n \rightarrow \infty}\left(\lambda_{n} / \mu_{n}\right)=1$ implies that $\liminf _{n \rightarrow \infty}\left(\lambda_{n} / \mu_{n}\right)=1>0$; that is, (11) $\Rightarrow(10)$.

Corollary 8. If $\lim _{n \rightarrow \infty}\left(\lambda_{n} / n\right)=1$ then

(i) if $\left(x_{k}\right) \in \ell_{\infty}$ and $x_{k} \rightarrow L\left(\widehat{S}_{\lambda}\right)$ then $x_{k} \rightarrow L[C, 1]$,

(ii) if $x_{k} \rightarrow L[C, 1]$ then $x_{k} \rightarrow L\left(\widehat{S}_{\lambda}\right)$.

\section{Competing Interests}

The authors declare that there is no conflict of interests regarding the publication of this paper.

\section{References}

[1] E. Savaš, "Strong almost convergence and almost $\lambda$-statistical convergence," Hokkaido Mathematical Journal, vol. 29, no. 3, pp. 531-536, 2000.

[2] L. Leindler, "Über die de la vallée-pousinsche summierbarkeit allgemeiner Orthogonalreihen," Acta Mathematica Academiae Scientiarum Hungaricae, vol. 16, pp. 375-387, 1965.

[3] M. Mursaleen, " $\lambda$-statistical convergence," Mathematica Slovaca, vol. 50, no. 1, pp. 111-115, 2000. 


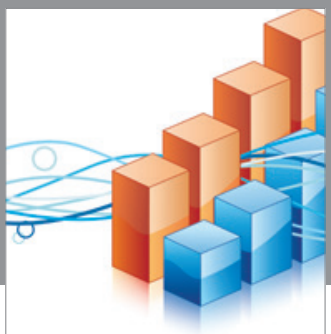

Advances in

Operations Research

vatem alat4

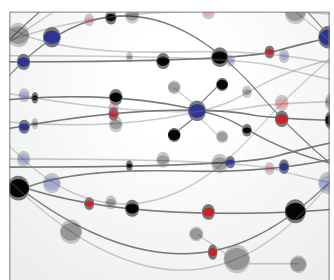

\section{The Scientific} World Journal
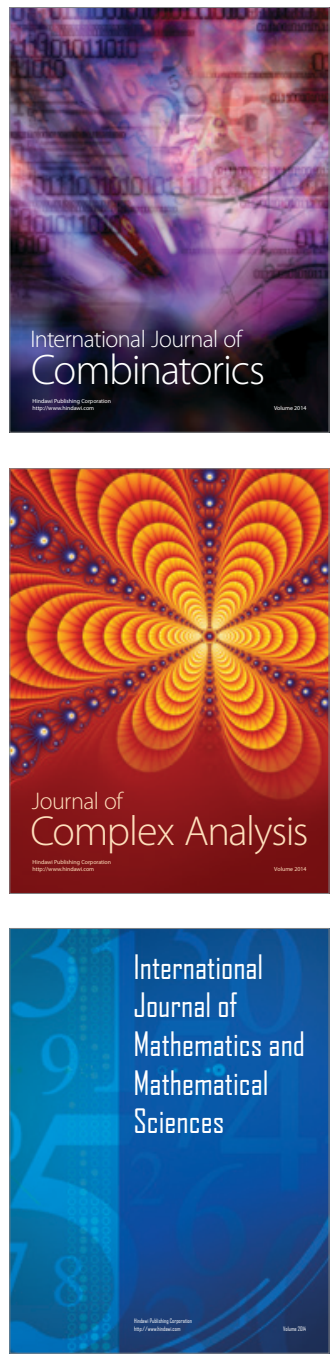
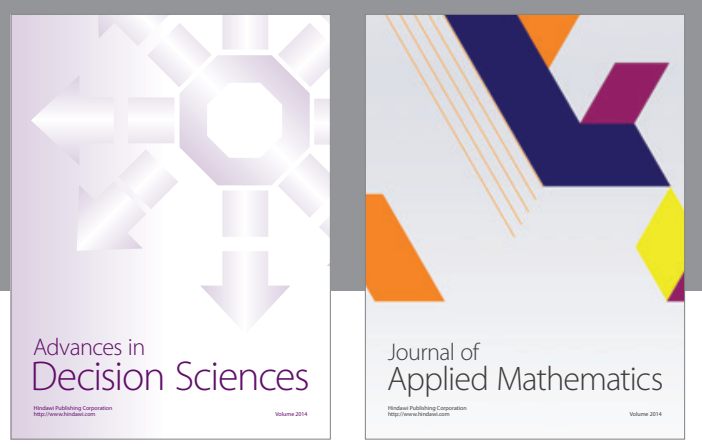

Algebra

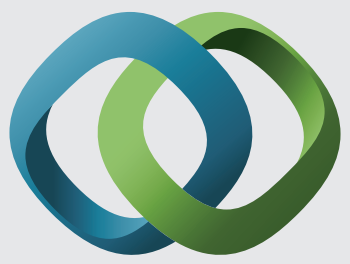

\section{Hindawi}

Submit your manuscripts at

http://www.hindawi.com
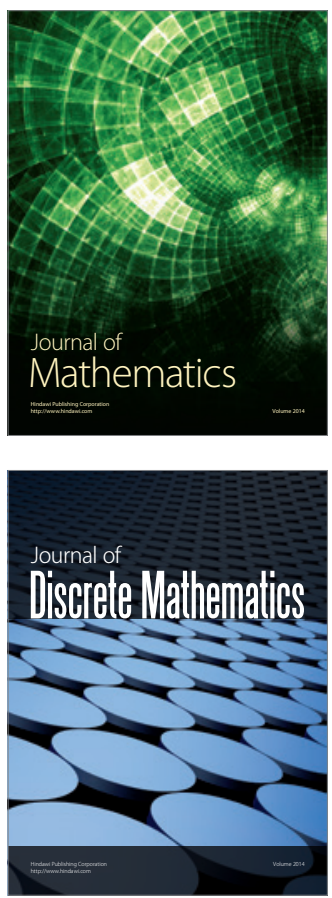

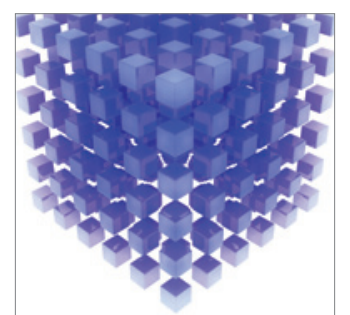

Mathematical Problems in Engineering
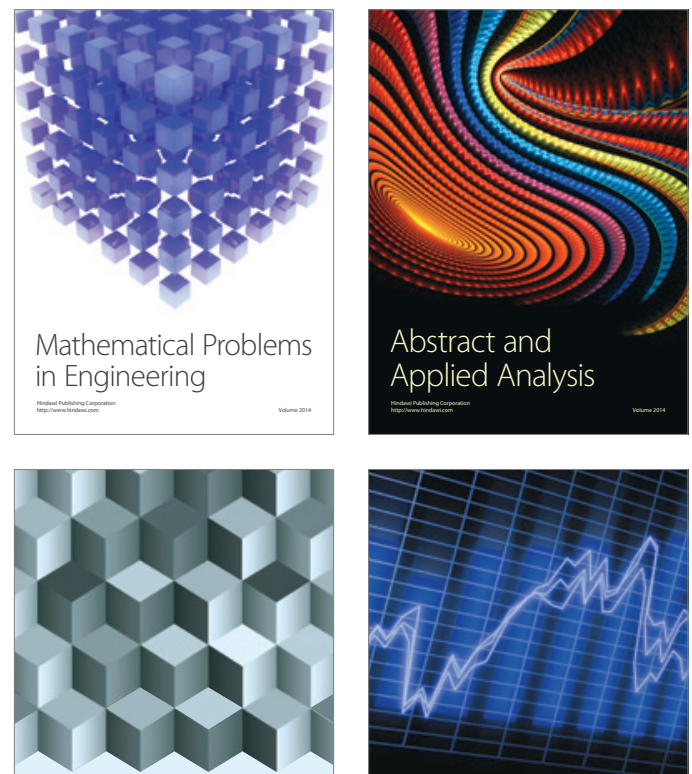

Journal of

Function Spaces

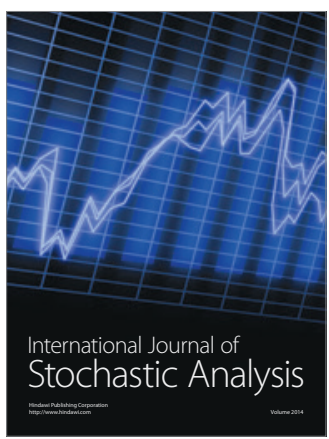

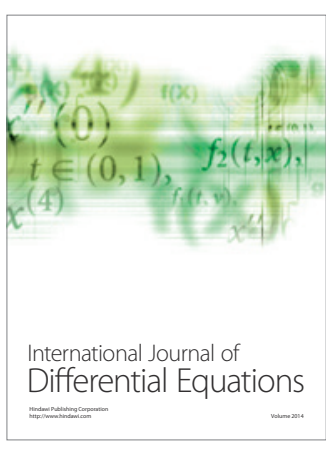
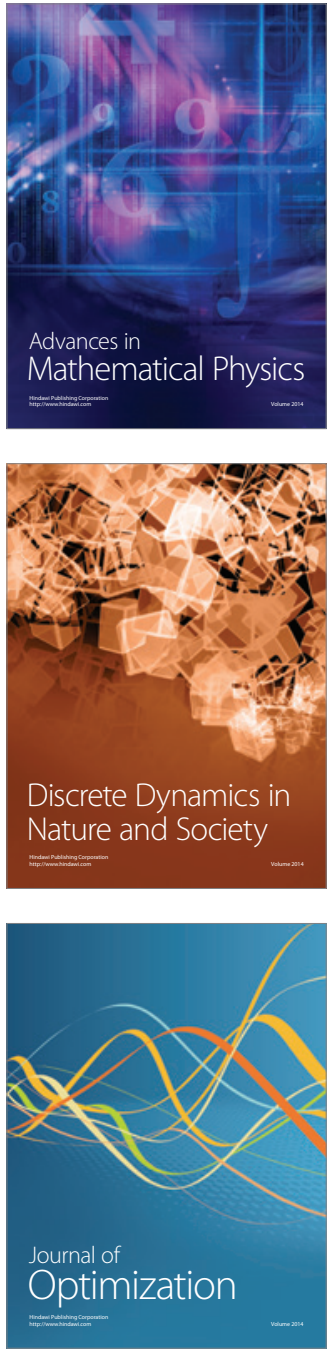